\title{
Maxillary Canine Ectopia and Maxillary Canine-Premolar Transposition are Associated with Deviations in the Maxilla
}

\author{
Louise Miltenburg Caspersen, Ib Jarle Christensen, and Inger Kjær
}

Department of Orthodontics, Institute of Dentistry, Faculty of Health Sciences, University of Copenhagen, Denmark

ABSTRACT The purpose was to analyze the direction of the infraorbital canal and the palatal width in cases with maxillary ectopic canines, all oriented horizontally and erupted labially, and with canine transposition and to compare findings with normal values. Eight anthropological human skulls, four with these horizontally oriented ectopic canines and four with canine-premolar transposition comprised the study. A radiopaque marker was placed in the infraorbital canal and frontal and profile radiographs were taken of each skull. Cephalometric measurements evaluated the canal direction (IOt angle). Interorbital $(\mathrm{IO})$ and palatal widths $(\mathrm{PW})$ were measured directly on the skulls. A general linear model was used for statistical analysis. Maxillary canine ectopia: $\operatorname{IOt}(\overline{\mathrm{x}}=8.54$; $95 \% \mathrm{CI}$ of -3.95 and 21.04; $\mathrm{P}=0.18)$ was larger, $\mathrm{PW}(\overline{\mathrm{x}}=$

A previous study on 42 normally developed anthropological skulls demonstrated that the direction of the infraorbital canal changes with age in the frontal view (Caspersen et al., 2009). This study also indicated that the direction of the infraorbital canal reflects the transversal growth of the maxilla.

Previous cephalometric studies have shown that the pterygoid canal and the mandibular canal are stable structures useful for superimposing of profile radiographs and therefore valuable when evaluating craniofacial growth patterns (Björk and Skieller, 1977, 1983). It is assumed that the infraorbital canal also is a stable structure during growth.

The infraorbital canal is located in the region of the maxilla, which has developed from the maxillary developmental field (Kjær, 2009). The palatal processes of the maxilla influencing the palatal width and the maxillary canines and premolars are also located within this field (Fig. 1). It is hypothesized that osseous deviations involving shape and width are expected in the maxilla in cases with dental deviations in the canine-premolar area. Such deviations are ectopic maxillary canine anomalies occurring with a frequency of 0.8 to 2.8\% (Aydin et al., 2004) and maxillary canine transpositions occurring with a frequency below $0.4 \%$ (Yilmaz et al., 2005). A dental transposition (or transmigration) occurs when teeth emerge in the wrong sequences in the dental arch, and the most common situation is when the maxillary canine emerges distal to the first premolar.
3.37; $95 \% \mathrm{CI}$ of 0.51 and $6.23 ; \mathrm{P}=0.022)$ was significantly smaller and $\mathrm{IO}(\overline{\mathrm{x}}=1.49 ; 95 \% \mathrm{CI}$ of -2.62 and 5.61; $\mathrm{P}=0.47)$ was also smaller. Maxillary canine-premolar transposition: IOt $(\bar{x}=17.27 ; 95 \% \mathrm{CI}$ of 4.78 and 29.76; $\mathrm{P}=0.008)$ and PW $(\bar{x}=3.34 ; 95 \% \mathrm{CI}$ of 0.48 and $6.21 ; \mathrm{P}=0.023)$ were significantly smaller and IO $(\bar{x}=2.94 ; 95 \%$ CI of -1.17 and 7.06; $\mathrm{P}=0.16$ ) was smaller, but not significantly. Eruptional deviations in the maxillary canines-premolars are associated with maxillary deviations expressed as the direction of the infraorbital canal and the transpalatal width. Accordingly, dental and osseous deviations within the maxillary developmental field are interrelated Dental Anthropology 2010;23(2):37-41.

The purpose of the present study was to analyze the direction of the infraorbital canal and the palatal width in cases either with (A) ectopic maxillary canines or (B) maxillary canine transpositions and to compare the findings with normal values. The ectopic canines were selected as possessing a horizontal orientation in the bone and these teeth had erupted labially into what would have been the subject's buccal vestibule.

\section{MATERIALS AND METHODS}

Skulls

Eight anthropological human skulls were analyzed, four with maxillary canine ectopia, all of which were oriented horizontally and had erupted labially ( 2 bilateral and 2 unilateral), and four with maxillary canine-premolar transposition ( 2 bilateral and 2 unilateral). In the ectopia cases, the canines were malpositioned and had failed to follow the normal eruption path (Fig. 2a). The skulls came from Björk's skull collection at the Department of Orthodontics, Copenhagen School of Dentistry, Denmark. The results from a previous study of 42 anthropological skulls from the same collection were used as normal

Correspondence to: Inger Kjær, Department of Orthodontics, School of Dentistry, Faculty of Health Sciences, University of Copenhagen, 20 Nørre Allé, DK-2200 Copenhagen N E-mail: ik@odont.ku.dk 


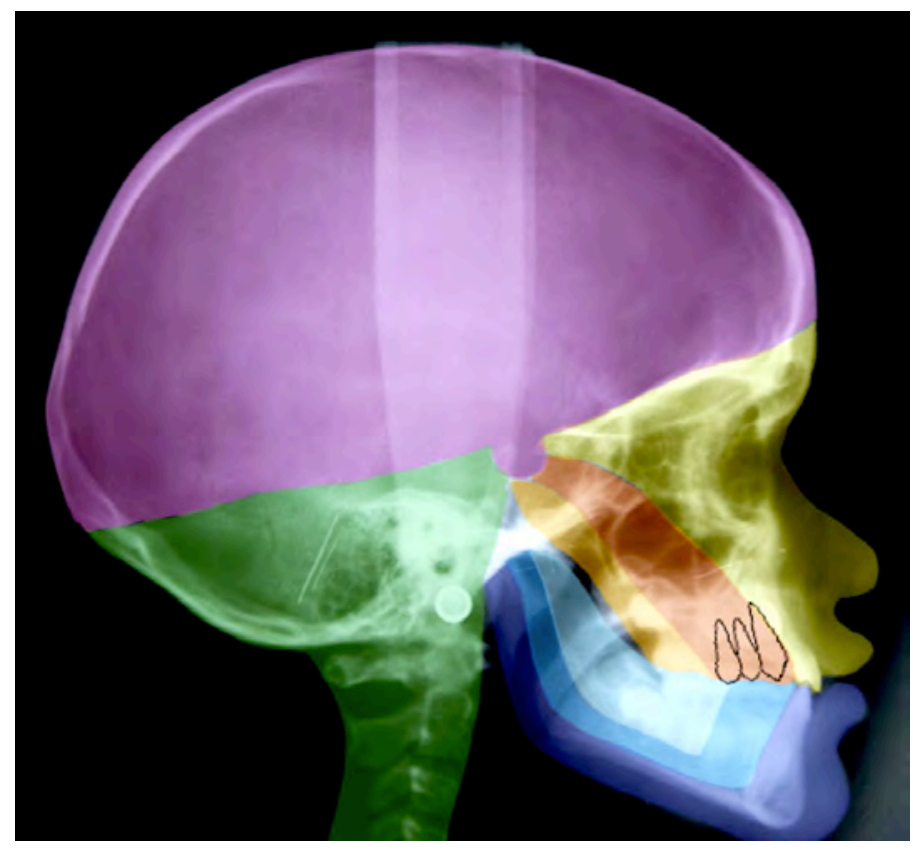

Fig. 1. On a profile radiograph of a 9 year-old girl different colored regions are marked indicating the embryological developmental fields defined by Kjær (2009). The maxillary field is the focus of the present study.

Yellow The frontonasal field

Red The maxillary field (canines and premolars are in this field)

Orange The palatal field

Blue Different fields in the mandible

Purple The theka field

Green The occipital field

[Color figure can be viewed in the electronic (PDF) version of the journal.]

controls (Caspersen et al., 2009).

\section{Registration of the infraorbital canal}

A radiopaque marker was placed in the infraorbital canal in the side where the maxillary canine ectopia (Fig. 2a) or the maxillary canine-premolar transposition (Fig. 2b) occurs. In the skulls with bilateral expressions, a marker was placed in both infraorbital canals.

\section{Radiography}

Frontal radiographs were taken of each skull as illustrated in Figure 3. The radiographs were taken at the Department of Orthodontics, School of Dentistry, Copenhagen, Denmark, in a Philips/Valmet BR 2002 cephalostat (Tagarno A/S, Horsens, Denmark) with a filmto-focus distance of $195 \mathrm{~cm}$. The linear enlargement was $8.3 \%$. The radiographic film used was LifeRay XDA Plus UTLG (Ferrania Technologies S.p.A., Cairo Montenotte, Italy). The films were exposed with $65-67 \mathrm{kv}$ and $5-7 \mathrm{~mA}$. The radiographs were taken with the skulls oriented in the
Frankfort Horizontal plane.

\section{Cephalometric analysis}

This analysis was performed according to the method developed by Caspersen et al. (2009). Tracing paper was placed on each frontal radiograph, and the outer contour of the skull, the orbital rim and piriform aperture were marked. Two lines were drawn: line 1 connecting the two bilateral orbital landmarks (lo) (Fig. 4) and line 2 expressing the direction of the infraorbital canal (Fig. 4). The bilateral orbital landmarks (lo) were defined according to Svanholt and Solow (1977). The angle between lines 1 and 2 (Fig. 4) was named the infraorbital transversal angle (IOt) (Caspersen et al., 2009).

\section{Direct skull measurements}

Two widths (one anterior and one posterior) were measured on the skulls according to Caspersen et al. (2009). The interorbital width (IO) was the length between the left and right infraorbital foramen; this is the anterior width. The palatal width (PW) was the maxillary cross-arch transversal palatal width (from first maxillary molar; left, to first maxillary molar, right); this is the posterior width.

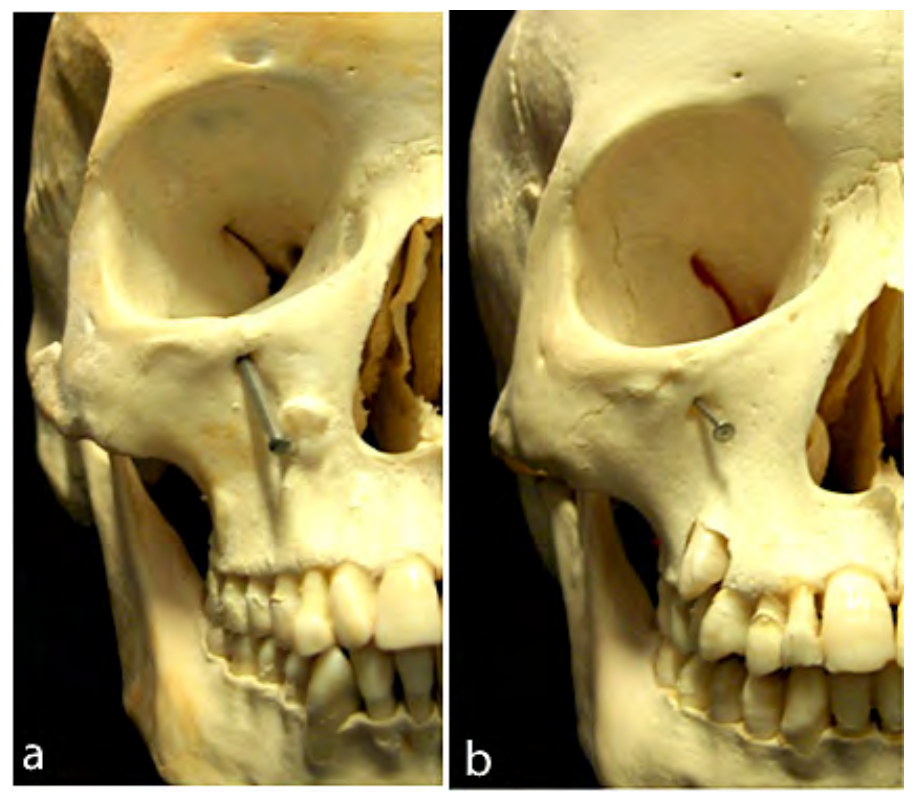

Fig. 2. Photographs of two anthropological skulls. (Left) Maxillary canine ectopia in the right side. The canine is oriented horizontally and has erupted labially. In this specimen, the permanent canine is lateral to the piriform plate, near the base of the nose, and the primary canine is still in occlusion. A radiopaque marker is placed in the infraorbital canal indicating the direction of the canal. (Right) Maxillary canine-premolar transposition in the right side. A radiopaque marker is placed in the infraorbital canal indicating the direction of the canal. 

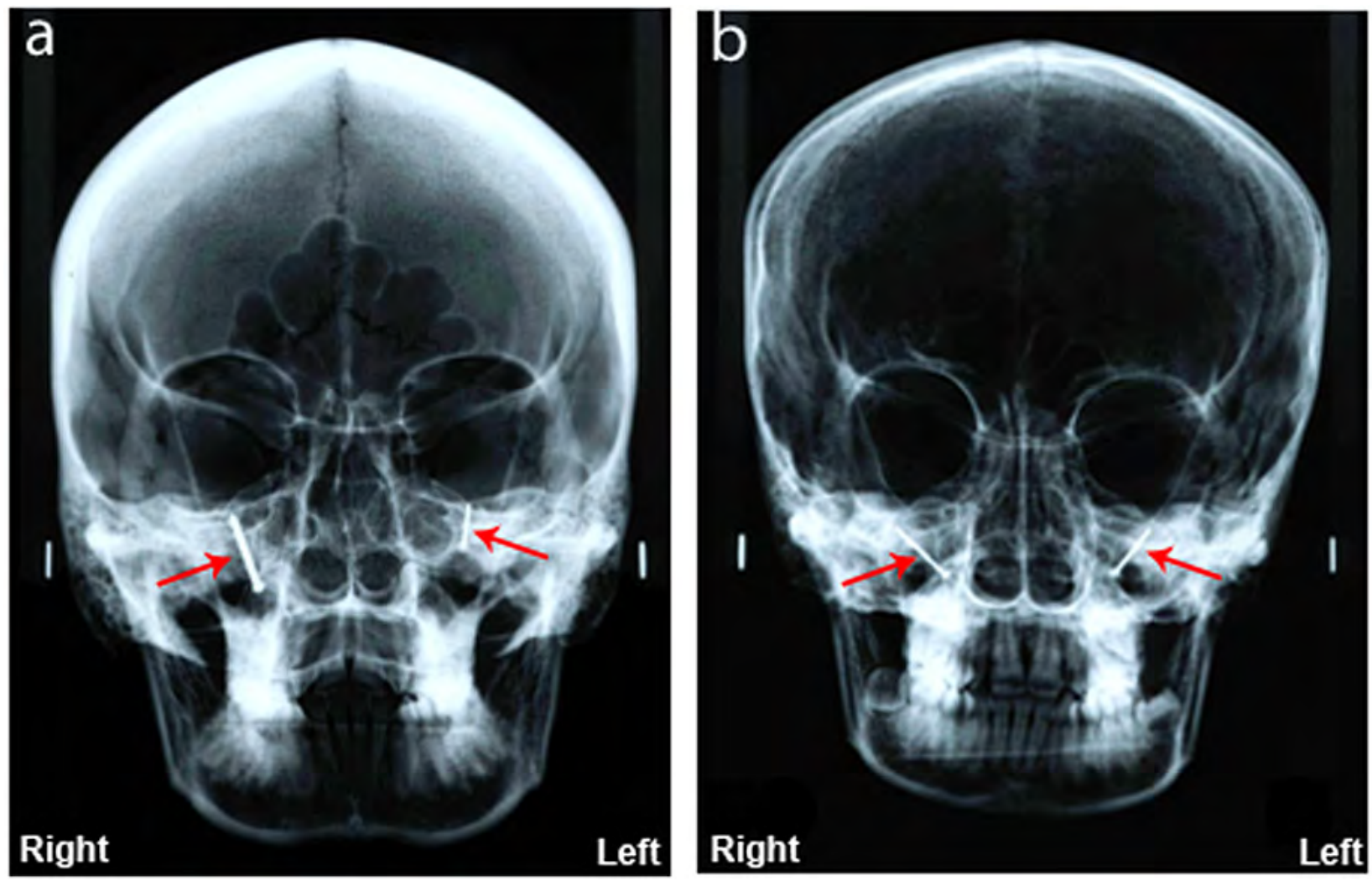

Fig. 3. Frontal radiographs of two anthropological skulls. (a) A skull with bilateral maxillary canine ectopia with a radiopaque marker in each infraorbital canal, visualized and marked by arrows. (b) A skull with bilateral maxillary canine-premolar transposition with a radiopaque marker in each infraorbital canal, visualized and marked by arrows.

\section{Statistical analysis}

A general linear model was used for statistical analysis of the three variables (IOt, PW, and IO). When the results from the ectopic and the transposition groups were compared with those of the control group, IOt, PW and IO were the dependent variables and the three different groups of skulls were the explanatory variables.

For all explanatory variables the mean level was subtracted. The results were presented by P-values and the estimates with 95\% Confidence Interval (CI). P-values less than $5 \%$ were considered significant. The analyses were performed using SAS (version 9.1, SAS Institute Inc., Cary, N.C., USA).

Further, in cases with bilateral registration the skulls were tested for differences between the sides. This test showed no significant difference $(\mathrm{P}=0.21$ for IOt; $\mathrm{P}=0.91$ for PW; $\mathrm{P}=0.98$ for $\mathrm{IO}$ ) and the mean value was used. This resulted in eight observations, namely four cases of maxillary labial canine ectopia (E) and four cases of maxillary canine-premolar transposition $(\mathrm{T})$.

\section{RESULTS}

The measurements of the three variables (IOt, IO, and PW) for the cases with canine ectopia (E) and caninepremolar transposition (T) are shown in Table 1. When all eight cases were evaluated a weak correlation was seen
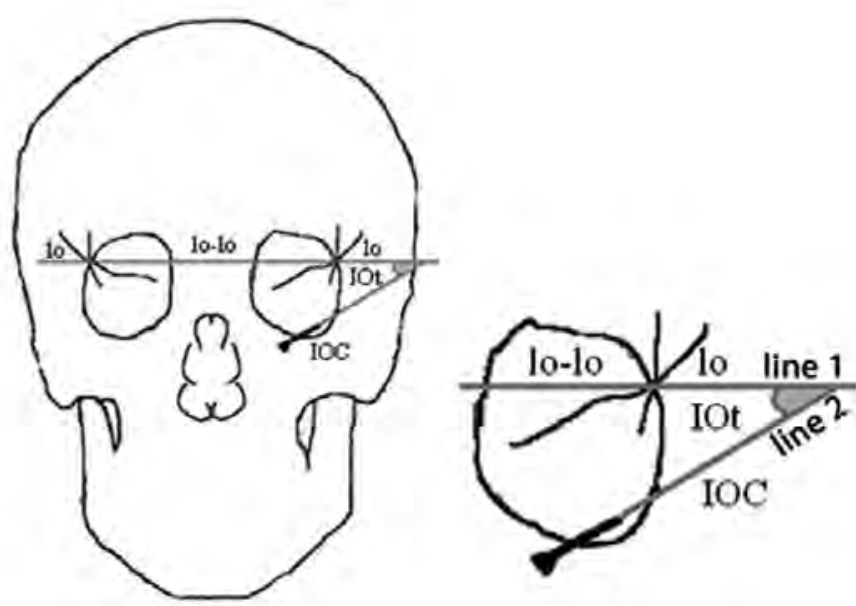

Fig. 4. The figure illustrates the tracing on the frontal cephalometric film of an anthropological cranium from a normal human adult. Line 1 represents the line through the contour of the bilateral orbital points (lo) and line 2 represents the direction of the infraorbital canal. The intersection between line 1 and line 2 forms the infraorbital transversal angle (IOt), which represents the inclination of the infraorbital canal. 
TABLE 1. Descriptive statistic of the three variables

\begin{tabular}{clllr}
\hline Group & Variable $^{\dagger}$ & $\mathrm{n}$ & mean & $\mathrm{sd}$ \\
\hline $\mathrm{E}$ & IOt (degrees) & 4 & 74.63 & 12.33 \\
$\mathrm{E}$ & PW (mm) & 4 & 34.05 & 1.11 \\
$\mathrm{E}$ & IO (mm) & 4 & 50.30 & 2.01 \\
$\mathrm{~T}$ & IOt (degrees) & 4 & 48.81 & 7.22 \\
$\mathrm{~T}$ & PW (mm) & 4 & 34.08 & 1.77 \\
$\mathrm{~T}$ & IO (mm) & 4 & 48.85 & 1.60 \\
\hline
\end{tabular}

tIOt: Infra orbital transversal angle

PW: Palatal width

IO: Inter orbital width.

The mean value and the standard deviation are given

for the four skulls with maxillary canine ectopia (E)

and for the four skulls with maxillary canine/premolar

transposition $(\mathrm{T})$.

For comparison normal values according to Caspersen $e t$ al. (2009) are:

IOt: mean 66.08 , sd 12.11

PW: mean 37.42, sd 2.85

IO: mean 51.79, sd 4.13

between IOt and IO $(\mathrm{P}=0.20)$, whereas no correlation was seen between IOt and PW or between PW and IO (Table 2).

Analysis showed that the infraorbital transversal angle (IOt) $(=8.54 ; 95 \% \mathrm{CI}$ of -3.95 and $21.04 ; \mathrm{P}=0.18)$ was larger and that the palatal width (PW) $(\overline{\mathrm{x}}=3.37 ; 95 \% \mathrm{CI}$ of 0.51 and $6.23 ; \mathrm{P}=0.022$ ) was significantly smaller than the mean value for the normal skulls. The interorbital width (IO) $(\bar{x}=1.49 ; 95 \% \mathrm{CI}$ of -2.62 and $5.61 ; \mathrm{P}=0.47)$ was also smaller.

The infraorbital transversal angle $(\mathrm{IOt})(\overline{\mathrm{x}}=17.27$; 95\% CI of 4.78 and 29.76; $\mathrm{P}=0.008$ ) and the palatal width $(\mathrm{PW})(\overline{\mathrm{x}}=3.34 ; 95 \% \mathrm{CI}$ of 0.48 and $6.21 ; \mathrm{P}=0.023)$ were significantly smaller compared with the normal skulls. Also, the interorbital width (IO) $(\bar{x}=2.94 ; 95 \%$ CI of -1.17

TABLE 2. Pearson correlations among the three variables

\begin{tabular}{lccc}
\hline Variable & IOt (degrees) & PW $(\mathrm{mm})$ & IO $(\mathrm{mm})$ \\
\hline IOt (degrees) & 1.000 & 0.13 & 0.51 \\
& & 0.77 & 0.20 \\
PW (mm) & 0.13 & 1.000 & 0.01 \\
& 0.77 & & 0.99 \\
IO (mm) & 0.51 & 0.01 & 1.000 \\
& 0.20 & 0.99 & \\
\hline
\end{tabular}

Correlation coefficients: $\mathrm{n}=4$; Prob $>$ Irl under $\mathrm{H}_{0}$ : Rho $=0$ Correlation between the three variables (IOt, PW and IO) in the 8 skulls with canine ectopia and canine/premolar transposition by Pearson correlation coefficients. The table shows a weak correlation between IOt and OI $(\mathrm{P}=0.20)$, whereas the other variables are not significantly correlated. and 7.06; $\mathrm{P}=0.16$ ) was smaller than the mean value in normal skulls, though not significantly.

This suggests that IOt and PW can be used as parameters expressing the maxillary complex in cases with maxillary canine ectopia and maxillary canine-premolar transposition. The infraorbital transversal angle (IOt) may be larger and the palatal width (PW) smaller in skulls with maxillary canine ectopia compared with normal skulls, whereas the infraorbital transversal angle (IOt) and the palatinal width are both smaller in skulls with maxillary canine-premolar transposition compared with normal skulls.

\section{DISCUSSION}

Two sorts of maxillary dental deviations were studied, namely (A) canine ectopia (where the canines are oriented horizontally and erupted labially) and (B) canine transposition. Analysis shows that both conditions are associated with skeletal deviations in the maxillary developmental field. This is a new observation. Meanwhile, the present study cannot explain the association between dental and osseous deviations. The question is whether the dental deviations are a result of regional developmental deviations in the field or whether the regional developmental deviations in the skeleton are a result of dental deviations. This question is closely associated with the etiology of the dental deviations, which cannot be explained by the present findings.

The etiology of canine ectopia and canine-premolar transposition is not known, but is assumed to be associated with multifactorial disorders involving genetic factors (Feichtinger et al., 1977; Peck et al., 1994). Whether a difference in the infraorbital canal direction exists between the type of ectopia described in this study where the canines are oriented horizontally and erupted labially and other types of canine ectopia cannot be determined from the data in this study. Also, inadequate space has been mentioned as a causative factor (Al-Nimri and Gharaibeh, 2005). Several studies have documented differences in the dentition in palatally and labially displaced ectopic canines (Chaushu et al., 2002; 2003; Sørensen et al., 2008).

The infraorbital canal and the maxillary canine are both located in the maxillary developmental field defined by Inger Kjær (2009). The deviated direction of the infraorbital canal discloses a deviation in the maxilla, which may influence the tooth eruption seen in cases with canine ectopia and canine-premolar transposition. A similar comparison of tooth development and bone development within a developmental field has previously been shown in the frontonasal field of the maxilla. The present material comprising four crania with ectopia and four crania with transposition may seem small, but considering the very low prevalence of both conditions (ectopia 0.8 to $2.8 \%$ and transposition below $0.4 \%$ ) the material represents a considerable population. Furthermore, among the types of ectopia, horizontally oriented and labially erupted canines are considered rare. 
Regarding the frontonasal field, the tooth deviation seen in SMMCI (Single Median Maxillary Central Incisor) has been associated with regional osseous deviations within the frontonasal field including the anterior wall of the sella turcica (Kjær et al., 2001; Becktor et al., 2001). The extension of the frontonasal field is demonstrated in Figure 1 (yellow color). It is recommended that the morphology of the sella turcica be investigated systematically in all cases of dental deviations in future studies.

The sagittal and vertical growth of the cranium can also affect the direction of the infraorbital canal, but in the present study only the frontal view was investigated because canine ectopia is often diagnosed in the frontal view on a panoramic radiograph. In the present study, the transverse width of the palate has been measured as an indicator of transverse growth in the mid-palatal suture. Under normal circumstances, palatal expansion is characterized by more extensive growth in the posterior region than anteriorly (Iseri and Solow, 1990). It seems that this usual pattern of growth does not occur in the cases evaluated in the present study.

The present study documents a statistically significant correlation between the direction of the infraorbital canal, maxillary morphology, and deviations in tooth eruption in the maxilla. It can be concluded that the maxillary dimensions are different in ectopia and transposition, and that the maxillary dimensions differ from normal findings.

\section{ACKNOWLEDGEMENTS}

The Faculty of Health Sciences, University of Copenhagen, Denmark, is acknowledged for founding. Helena Schatz is acknowledged for professional assistance with the radiographic procedure. Maria Kvetny is acknowledged for linguistic support and manuscript preparation.

\section{LITERATURE CITED}

Al-Nimri K, Gharaibeh T. 2005. Space conditions and dental and occlusal features in patients with palatally impacted maxillary canines: an aetiological study. Eur J Orthod 27:461-465.

Aydin U, Yilmaz HH, Yildirim D. 2004. Incidence of canine impaction and transmigration in a patient population. Dentomaxillofac Radiol 33:164-169.

Becktor KB, Sverrild L, Pallisgaard C, Burhøj J, Kjær I. 2001. Eruption of the central incisor, the intermaxillary suture, and maxillary growth in patients with a single median maxillary central incisor, SMMCI. Acta Odontol
Scand 59:361-366.

Björk A, Skieller V. 1977. Growth of the maxilla in three dimensions as revealed radiographically by the implant method. Br J Orthod 4:53-64.

Björk A, Skieller V. 1983. Normal and abnormal growth of the mandible: a synthesis of longitudinal cephalometric implant studies over a period of 25 years. Eur J Orthod 5:1-46.

Caspersen LM, Christensen IJ, Kjær I. 2009. Inclination of the infraorbital canal studied on dry skulls expresses the maxillary growth pattern - a new contribution to the understanding of change in inclination of ectopic canines during puberty. Acta Odontol Scand 17:1-5.

Chaushu S, Sharabi S, Becker A. 2002. Dental morphologic characteristics of normal versus delayed developing dentitions with palatally displaced canines. Am J Orthod Dentofacial Orthop 121:339-346.

Chaushu S, Sharabi S, Becker A. 2003. Tooth size in dentitions with buccal canine ectopia. Eur J Orthod 25:485-491.

Feichtinger C, Rossiwall B, Wunderer H. 1977. Canine transposition as autosomal recessive trait in an inbred kindred. J Dent Res 56:1449-1452.

Iseri H, Solow B. 1990. Growth displacement of the maxilla in girls studied by implant method. Eur J Orthod 12:389-398.

Kjær I. 2010. Orthodontics and foetal pathology: a personal view on craniofacial patterning. Eur J Orthod 32:40-47.

Kjær I, Becktor KB, Lisson J, Gormsen C, Russell BG. 2001. Face, palate and craniofacial morphology in patients with a solitary median maxillary central incisor. Eur J Orthod 23:63-73.

Peck S, Peck L, Kataja M. 1994. The palatally displaced canine as a dental anomaly of genetic origin. Angle Orthod 64:249-256.

Svanholt P, Solow B. 1977. Assessment of midline discrepancies in the postero-anterior cephalometric radiograph. Trans Eur Orthod Soc 25:261-268.

Sørensen HB, Artmann L, Larsen HJ, Kjær I. 2008. Radiographic assessment of dental anomalies in patients with ectopic maxillary canines. Int J Paediatr Dent 19:108-114.

Yilmaz HH, Türkkahraman H, Sayin MÖ. 2005. Prevalence of tooth transpositions and associated dental anomalies in a Turkish population. Dentomaxillofac Radiol 34:3235. 